\title{
gु
}

\section{Nuclear Force Imprints Revealed on the Elastic Scattering of Protons with ${ }^{10} \mathrm{C}$}

\author{
A. Kumar, ${ }^{1}$ R. Kanungo, ${ }^{1 *}$ A. Calci, ${ }^{2}$ P. Navrátill, ${ }^{2 \dagger}$ A. Sanetullaev, ${ }^{1,2}$ M. Alcorta, ${ }^{2}$ V. Bildstein, ${ }^{3}$ G. Christian, ${ }^{2}$ \\ B. Davids, ${ }^{2}$ J. Dohet-Eraly, ${ }^{2,4}$ J. Fallis, ${ }^{2}$ A. T. Gallant, ${ }^{2}$ G. Hackman, ${ }^{2}$ B. Hadinia, ${ }^{3}$ G. Hupin, ${ }^{5,6}$ S. Ishimoto, ${ }^{7}$ \\ R. Krücken, ${ }^{2,8}$ A. T. Laffoley, ${ }^{3}$ J. Lighthall, ${ }^{2}$ D. Miller, ${ }^{2}$ S. Quaglioni, ${ }^{9}$ J. S. Randhawa, ${ }^{1}$ E. T. Rand, ${ }^{3}$ \\ A. Rojas, ${ }^{2}$ R. Roth, ${ }^{10}$ A. Shotter, ${ }^{11}$ J. Tanaka, ${ }^{12}$ I. Tanihata, ${ }^{12,13}$ and C. Unsworth ${ }^{2}$ \\ ${ }^{1}$ Astronomy and Physics Department, Saint Mary's University, Halifax, Nova Scotia B3H 3C3, Canada \\ ${ }^{2}$ TRIUMF, Vancouver, British Columbia V6T2A3, Canada \\ ${ }^{3}$ Department of Physics, University of Guelph, Guelph, Ontario NIG 2W1, Canada \\ ${ }^{4}$ Istituto Nazionale di Fisica Nucleare, Sezione di Pisa, Largo B. Pontecorvo 3, I-56127 Pisa, Italy \\ ${ }^{5}$ Institut de Physique Nucléaire, Université Paris-Sud, IN2P3/CNRS, F-91406 Orsay Cedex, France \\ ${ }^{6}$ CEA, DAM, DIF, F-91297 Arpajon, France \\ ${ }^{7}$ High Energy Accelerator Research Organization (KEK), Ibaraki 305-0801, Japan \\ ${ }^{8}$ Department of Physics and Astronomy, University of British Columbia, Vancouver, British Columbia V6T 1Z1, Canada \\ ${ }^{9}$ Lawrence Livermore National Laboratory, P.O. Box 808, L-414, Livermore, California 94551, USA \\ ${ }^{10}$ Institut fur Kernphysik, Technische Universitat Darmstadt, 64289 Darmstadt, Germany \\ ${ }^{11}$ School of Physics and Astronomy, University of Edinburgh, JCMB, Mayfield Road, Edinburgh EH9 3JZ, United Kingdom \\ ${ }^{12}$ RCNP, Osaka University, Mihogaoka, Ibaraki, Osaka 567 0047, Japan \\ ${ }^{13}$ School of Physics and Nuclear Energy Engineering and IRCNPC, Beihang University, Beijing 100191, China
}

(Received 8 March 2017; published 28 June 2017)

\begin{abstract}
How does nature hold together protons and neutrons to form the wide variety of complex nuclei in the Universe? Describing many-nucleon systems from the fundamental theory of quantum chromodynamics has been the greatest challenge in answering this question. The chiral effective field theory description of the nuclear force now makes this possible but requires certain parameters that are not uniquely determined. Defining the nuclear force needs identification of observables sensitive to the different parametrizations. From a measurement of proton elastic scattering on ${ }^{10} \mathrm{C}$ at TRIUMF and $a b$ initio nuclear reaction calculations, we show that the shape and magnitude of the measured differential cross section is strongly sensitive to the nuclear force prescription.
\end{abstract}

DOI: 10.1103/PhysRevLett.118.262502

Understanding the strong nuclear force is of fundamental importance to decipher nature's way of building visible matter in our Universe. Yet, more than a century after the discovery of the nucleus, our knowledge of the nuclear force is still incomplete. The formulation by Weinberg of chiral effective field theory [1] enabled a major breakthrough in arriving at a fundamental understanding of the low-energy nuclear interactions of protons and neutrons, by forging the missing link with quantum chromodynamics. However, the question of how to best implement the theory and constrain it with experimental data remains an active topic of research, and has already led to several parametrizations of the nuclear force [2-6]. It is, therefore, important to identify experimental observables that are sensitive to different parametrizations of the chiral forces in order to reach a definitive description of the nuclear force. The study of many-nucleon systems enables a more complete understanding of the nuclear force. In particular, proton-rich and neutron-rich nuclei located at the edges of nuclear stability (drip lines) can amplify less-constrained features of the nuclear force, such as its dependence on the proton-neutron asymmetry. However, there is a lack of experimental data on the properties of these systems.
Among the properties of the drip-line nuclei, we hypothesize in this work that the nucleon-nucleus scattering differential cross section is highly sensitive to the details of the nuclear force and, hence, can be used for constraining it. Indeed, it should reveal both the spectroscopic properties of the reacting system, such as phase shifts and their interference, as well as the effect of exotic nucleon distributions. This confluence brings a greater selectivity in the elastic scattering differential cross section than is possible by independently investigating resonance energies, binding energies, or radii. The observations reported here show that the shape and magnitude of the elastic scattering angular distribution places stringent constraints on the chiral interactions, while a study of resonance energies alone could lead to incomplete and/or misleading conclusions. The study of elastic scattering for drip-line nuclei is, however, challenging because of the low-beam intensities and formulation of the $a b$ initio structure and reaction theory.

We report the first investigation probing the nuclear force through proton elastic scattering from ${ }^{10} \mathrm{C}$, located at the proton drip line. This is an ideal system to test the effect of the nuclear force. This is because, firstly, the very existence 
of bound ${ }^{10} \mathrm{C}$ whose isotonic neighbors ${ }^{9} \mathrm{~B},{ }^{8} \mathrm{Be}$, and ${ }^{11} \mathrm{~N}$ are unbound, is a testament of the complicated strong interaction. Secondly, $a b$ initio Green's function Monte Carlo [7] and no-core shell model [8,9] calculations have shown the three-nucleon force to be important for explaining the structure of mass number $A=10$ nuclei. Recent advances in $a b$ initio nuclear reaction theory now allow us to compute the ${ }^{10} \mathrm{C}(p, p)$ scattering cross section based on chiral forces. Thirdly, with the low-energy reaccelerated beam available at TRIUMF, our investigation was carried out at low center-of-mass energies of 2.1-4.4 MeV for $p+{ }^{10} \mathrm{C}$, since here the low-level density of the composite (unbound) nucleus ${ }^{11} \mathrm{~N}$ minimizes the number of phase shifts influencing the diffraction pattern, and hence facilitates the identification of nuclear force effects more transparently than what is possible with stable nuclei. Furthermore, no transfer reaction channels are open at low energy for this system, thereby simplifying the $a b$ initio reaction calculation.

The experiment was performed in inverse kinematics at the ISAC rare isotope beam facility at TRIUMF $[10,11]$ by bombarding a proton target with a ${ }^{10} \mathrm{C}$ beam. The beam with an average intensity of 2000 particles per second reaccelerated using ISAC-II superconducting linear accelerator $[10,12]$, impinged on a solid hydrogen target at the IRIS reaction spectroscopy station [13]. A schematic of the setup is shown in Fig. 1. Energy loss measured in a lowpressure ionization chamber allowed for clean identification of ${ }^{10} \mathrm{C}$ from the ${ }^{10} \mathrm{~B}$ contaminant. The beam energies at midtarget were $4.54 \mathrm{~A} \mathrm{MeV}$ and $4.82 \mathrm{~A} \mathrm{MeV}$, corresponding to $p+{ }^{10} \mathrm{C}$ center-of-mass energies of $E_{\text {c.m. }}=4.15$ and 4.4 $\mathrm{MeV}$, respectively. These energies were chosen to be around the location of the $5 / 2^{+}$and $3 / 2^{-}$resonances in the ${ }^{11} \mathrm{~N}$ compound system $\left(={ }^{10} \mathrm{C}+p\right)$ because preliminary calculations suggested that variation of the nuclear force alters the $D_{5 / 2}$ and $P_{3 / 2}$ phase shifts and hence the cross sections significantly. Our selected energies were chosen to be below and above the $3 / 2^{-}$resonance, which is placed at 4.35(3) $\mathrm{MeV}$ in the evaluation in Ref. [14]. We note here, however, that conflicting experimental data exist on this

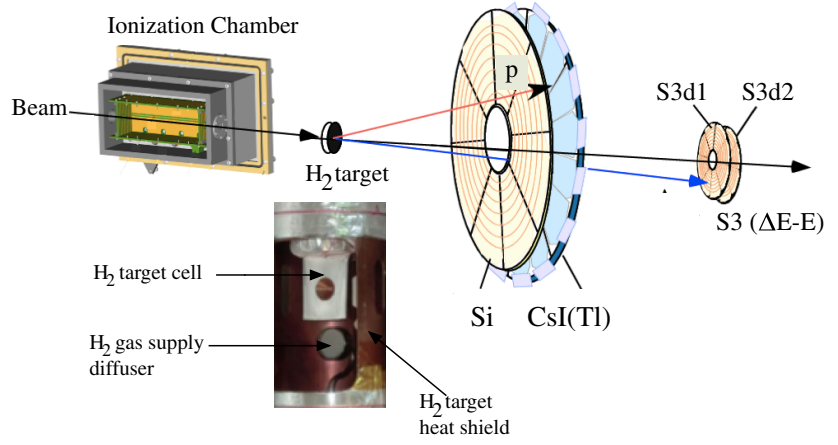

FIG. 1. Schematic view of the experiment setup at the IRIS reaction spectroscopy station. resonance position. Reference [15] places the $3 / 2^{-}$resonance at 4.56(1) MeV, which is higher than both the beam energies, in which case the cross sections at the two measured energies may be similar.

The scattered protons were identified using the correlation between energy loss in an annular array of segmented silicon detectors and the remaining energy deposited in $\mathrm{CsI}(\mathrm{Tl})$ detectors covering angles $\theta_{\mathrm{lab}} \sim 26^{\circ}-52^{\circ}$. The selected proton events show a very clear locus of elastic scattering [Fig. 2(a)]. The inelastic scattering locus is only slightly visible around $\theta_{\mathrm{lab}} \sim 26^{\circ}-28^{\circ}$, as most of this channel occurs at smaller $\theta_{\mathrm{lab}}$ and was hence outside the detector coverage. The excitation energy spectrum of ${ }^{10} \mathrm{C}$ [Fig. 2(b)] was reconstructed from the measured energies and scattering angles of the protons using the missing mass technique. A small background, seen under the elastic peak, estimated by a linear fit to be $\sim 1 \%-3 \%$, was subtracted to obtain the elastic scattering cross sections at the different scattering angles.
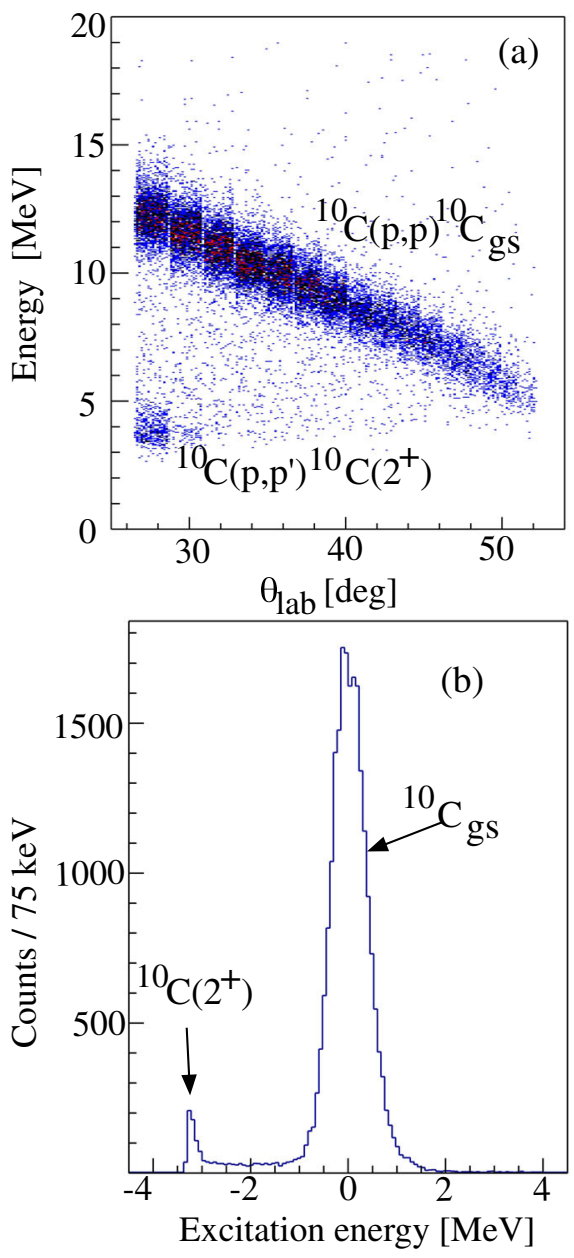

FIG. 2. (a) The measured kinematic loci, proton energy as a function of proton scattering angle, for ${ }^{10} \mathrm{C}(p, p){ }^{10} \mathrm{C}_{g s}$ at $E_{\text {c.m. }}=4.15 \mathrm{MeV}$. (b) Measured excitation energy spectrum of ${ }^{10} \mathrm{C}$. 
The scattered ${ }^{10} \mathrm{C}$ was detected by double-sided segmented silicon strip detectors (S3). The solid $\mathrm{H}_{2}$ (proton) target was formed on a $5.4 \mu \mathrm{m} \mathrm{Ag}$ foil backing. The energy of the ${ }^{10} \mathrm{C}+\mathrm{Ag}$ elastic scattering peak, measured with and without $\mathrm{H}_{2}$, was used to determine the $\mathrm{H}_{2}$ target thickness from the energy loss of scattered ${ }^{10} \mathrm{C}$ through $\mathrm{H}_{2}$. The ${ }^{10} \mathrm{C}+\mathrm{Ag}$ scattering with the presence of $\mathrm{H}_{2}$ was measured simultaneously with the ${ }^{10} \mathrm{C}(p, p)$ reaction continuously throughout the experiment; hence, the target thickness at each instant was accurately known. The average target thickness was $\sim 80 \mu \mathrm{m}$. The number of incident beam particles was counted using the ionization chamber. Since the beam intensity and target thickness were measured continuously during the experiment, the absolute magnitude of the cross section is well determined. See Supplemental Material for experiment details [16].

The measured differential cross section for ${ }^{10} \mathrm{C}(p, p)$ in the center-of-mass frame is shown in Fig. 3. The experimental data contain both statistical and systematic uncertainties. The systematic uncertainties are as follows: $5 \%$ from the target thickness, $5 \%$ from determination of the detection efficiency, and $4 \%$ from the beam contamination, which were added in quadrature. The cross sections have similar shape and magnitude at the two different beam energies. $A b$ initio reaction theory calculations with three different choices of the nuclear force are shown by the curves.

Our theoretical description of the ${ }^{10} \mathrm{C}(p, p)$ scattering is based on the ab initio no-core shell model with continuum

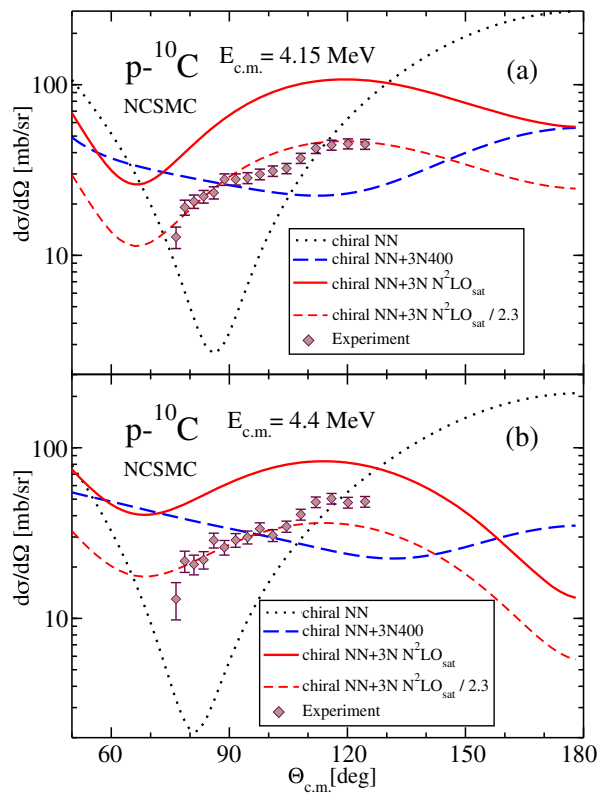

FIG. 3. Measured differential cross section for ${ }^{10} \mathrm{C}(p, p){ }^{10} \mathrm{C}_{g s}$ at (a) $E_{\text {c.m. }}=4.15$ and (b) $E_{\text {c.m. }}=4.4 \mathrm{MeV}$. The curves are $a b$ initio theory calculations. The black dotted, blue long dashed, and red solid curves are with the chiral $N N, N N+3 N 400$, $\mathrm{N}^{2} \mathrm{LO}_{\text {sat }}$ interactions, respectively. The red dashed curve is the $\mathrm{N}^{2} \mathrm{LO}_{\text {sat }}$ calculation scaled down by a factor of 2.3 .
(NCSMC) [37-39]. This approach describes the reacting system using a basis expansion with two key components: one describing all nucleons close together, forming the ${ }^{11} \mathrm{~N}$ nucleus, and a second one describing the separated proton and ${ }^{10} \mathrm{C}$ clusters. The former part utilizes a squareintegrable basis expansion treating all 11 nucleons on the same footing. The latter part factorizes the wave function into products of ${ }^{10} \mathrm{C}$ and proton components and their relative motion with proper scattering boundary conditions. The chiral two-nucleon $(N N)$ and three-nucleon $(3 N)$ forces served as input for the NCSMC calculations. See Supplemental Material for more details on the calculation [16].

In chiral effective field theory, the dynamics due to unresolved physics, i.e., degrees of freedom other than nucleons and pions, is accounted for by contact interactions with parameters calculable in principle from QCD although presently fitted to experimental data. In particular, the $N N$ interaction is tuned to nucleon-nucleon phase shifts and the deuteron properties. Traditionally, the fit of the $N N$ parameters was performed first [2], and the $3 \mathrm{~N}$ parameters were adjusted to ${ }^{3} \mathrm{H} /{ }^{3} \mathrm{He}[40,41]$ and sometimes also ${ }^{4} \mathrm{He}$ data [4] in a second step. The $N N+3 N 400$ force ( $N N$ from Ref. [2] and $3 N$ from Ref. [4] with cutoff of $400 \mathrm{MeV}$ ) pertains to this family of interactions and describes well the binding energy of the $\mathrm{O}, \mathrm{N}$, and $\mathrm{F}$ isotopes $[42,43]$. Recently, a simultaneous $N N+3 N$ fit at next-to-next-leading-order $\left(\mathrm{N}^{2} \mathrm{LO}\right)$ has been performed using not just the two-nucleon and $A=3,4$ data, but also binding energies of ${ }^{14} \mathrm{C}$ and ${ }^{16,22,24,25} \mathrm{O}$ as well as charge radii of ${ }^{14} \mathrm{C}$ and ${ }^{16} \mathrm{O}$ [5]. The resulting interaction, named $\mathrm{N}^{2} \mathrm{LO}_{\text {sat }}$, successfully describes the saturation of infinite nuclear matter [5], the proton radius of the stable nucleus ${ }^{48} \mathrm{Ca}$ [44], and the proton and matter radii of neutron-rich carbon isotopes [45].

We test these two parametrizations of chiral $N N+3 N$ forces and, further, investigate the impact of the chiral $3 \mathrm{~N}$ force by comparing them with a chiral $N N$ interaction alone. Figure 3 shows that the shape and magnitude of the angular distribution is strongly influenced by the nuclear force prescription. The results obtained with the $N N$ interaction from Ref. [2] (black dotted curves) show a strong dip in the cross section at $\theta_{\text {c.m. }} \sim 80^{\circ}$, while no such feature is observed in the data. The addition of the $3 N$ force with a momentum cutoff of $400 \mathrm{MeV}[6](N N+3 N 400$, blue long dashed curves) produces a much different shape. This shows the strong influence of the three-nucleon interaction on the angular distribution. While it improves the overall agreement with the data, the addition of the $3 \mathrm{~N}$ force in the $N N+3 N 400$ interaction clearly still does not explain the observed angular distribution characteristics. Meanwhile, with the $\mathrm{N}^{2} \mathrm{LO}_{\text {sat }} N N+3 N$ interaction (red solid curves), the predicted shape of the angular distribution is in very good agreement with the data, as evidenced by the scaled result (red dashed curves). The magnitude of the cross section is higher than the data, which, as discussed 
below, reflects effects of $S_{1 / 2}$ phase shift(s) and, hence, places further constraint on the force prescription.

Compared to calculations with the $N N$ interaction, the $\mathrm{N}^{2} \mathrm{LO}_{\text {sat }}$ and $N N+3 N 400$ forces result in only a small $\sim 1 \%$ effect in static properties, such as the proton and matter radii of ${ }^{10} \mathrm{C}$. While the binding energy predicted by $N N+3 N 400$ is lower by $\sim 12 \%$ than the predictions from $\mathrm{N}^{2} \mathrm{LO}_{\text {sat }}$, the latter is $\sim 3 \%$ higher than the experimental value; thus, a binding energy-based distinction of the different forces is not straightforward. However, here we find a strong constraint on the nuclear force emerging from the dramatic change of the angular distribution shape (Fig. 3). The chi-square $\left(\chi^{2}\right)$ values of scaled cross sections show this clearly. For $E_{\text {c.m. }}=4.15 \mathrm{MeV}$, the best fit $\chi^{2}=65.5$ for the $N N, 25.9$ for the $N N+3 N 400$, and 1.7 for the $\mathrm{N}^{2} \mathrm{LO}_{\text {sat }}$ interactions (after its scaling), making $\mathrm{N}^{2} \mathrm{LO}_{\text {sat }}$ the best selection among the three. Additionally, the failure of the $N N+3 N 400$ interaction to reproduce the angular distribution despite good predictions of the binding energies of the oxygen isotopes $[42,43]$ shows the limited selectivity of binding energies in differentiating among the nuclear force models. The magnitude of the cross section, however, shows the deficiencies of the $\mathrm{N}^{2} \mathrm{LO}_{\text {sat }}$ interaction.

Calculated phase shifts with the three interactions are shown in Fig. 4. The resonance energies from $R$-matrix analysis are listed in Table I. In the energy region of the experiment (vertical lines in Fig. 4), the shape of the angular distribution is dominated by the $3 / 2^{-}$and the $5 / 2^{+}$phase shifts. The $5 / 2^{+}$resonance couples strongly

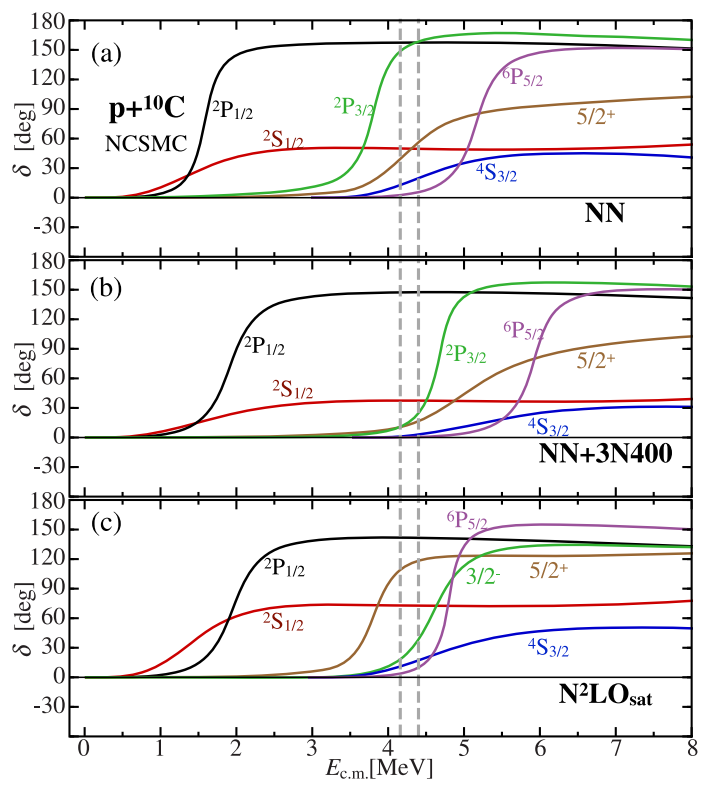

FIG. 4. Calculated $p+{ }^{10} \mathrm{C}$ phase shifts (eigenphase shifts for $5 / 2^{+}$and $\left.\mathrm{N}^{2} \mathrm{LO}_{\text {sat }} 3 / 2^{-}\right)$. Ab initio NCSMC results obtained using chiral $N N\left(\right.$ a), chiral $N N+3 N 400$ (b), and chiral $\mathrm{N}^{2} \mathrm{LO}_{\text {sat }}$ (c) are compared. The vertical dashed lines show the energies where the experiment was performed. the ${ }^{2} D_{5 / 2}\left[{ }^{10} \mathrm{C}\left(0^{+}\right)\right]$and ${ }^{6} S_{5 / 2}\left[{ }^{10} \mathrm{C}\left(2_{1}^{+}\right)\right]$partial waves. Therefore, we present the $5 / 2^{+}$eigenphase shifts. The other shown resonances are dominated by a single (shown) partial wave with the exception of the $\mathrm{N}^{2} \mathrm{LO}_{\text {sat }} 3 / 2^{-}$that couples ${ }^{2} P_{3 / 2}\left[{ }^{10} \mathrm{C}\left(0^{+}\right)\right]$and ${ }^{6} P_{3 / 2}\left[{ }^{10} \mathrm{C}\left(2_{1}^{+}\right)\right]$partial waves.

We observe that the $5 / 2^{+}$and $3 / 2^{-}$resonances are placed differently in the three calculations. Using the chiral $N N$ interaction alone [Fig. 4(a)], the $3 / 2^{-}$resonance is below the $5 / 2^{+}$one and below the ${ }^{10} \mathrm{C}(p, p)$ experimental region. Switching on the chiral $3 N$ force in the $N N+$ $3 N 400$ calculation [Fig. 4(b)] the two resonances are almost degenerate and slightly above the region of measurement. With the chiral $\mathrm{N}^{2} \mathrm{LO}_{\text {sat }}(N N+3 N)$ interaction, the $5 / 2^{+}$resonance is below the $3 / 2^{-}$one and below the energy region where the ${ }^{10} \mathrm{C}(p, p)$ measurements were performed [Fig. 4(c)]. Using only the $\mathrm{N}^{2} \mathrm{LO}_{\text {sat }}$ interaction, the ordering of the $5 / 2^{+}$and the $3 / 2^{-}$is in qualitative agreement with the established ordering of the isospin analog resonances in the mirror ${ }^{11} \mathrm{Be}$ nucleus [14,46].

A comparison of the computed $3 / 2^{-}$and $5 / 2^{+}$resonance properties (Table I) to the evaluated data therefore could erroneously lead one to believe that the $\mathrm{N}^{2} \mathrm{LO}_{\text {sat }}$ interaction works almost perfectly. However, the magnitude of our measured cross section is overestimated by the $\mathrm{N}^{2} \mathrm{LO}_{\text {sat }}$ calculations. Hence, it should be emphasized that the present experiment tests the nuclear force more strictly than a straight comparison of energies and widths of the resonances. This is because the differential cross section also receives contributions from phase shifts in other partial waves; in this case, in particular from the ${ }^{2} S_{1 / 2}$ that contributes only to the magnitude of the cross section and is much more pronounced in the $\mathrm{N}^{2} \mathrm{LO}_{\text {sat }}$ calculation.

The present reaction calculation does not include the ${ }^{9} \mathrm{Be}+2 p$ breakup channel, which lies just a few hundred $\mathrm{keV}$ below the energy of the experiment. This omission contributes only a small part to the overprediction of the data by the $\mathrm{N}^{2} \mathrm{LO}_{\text {sat }}$ interaction. An estimate of this is obtained from our calculated ${ }^{10} \mathrm{C}\left(p, p^{\prime}\right){ }^{10} \mathrm{C}\left(2^{+}\right)$inelastic cross section and is only a few $\mathrm{mb} / \mathrm{sr}$ at the same relative energy. Therefore, given the reasonable convergence of our calculations, this shows that the $\mathrm{N}^{2} \mathrm{LO}_{\text {sat }}$ interaction,

TABLE I. Energies $\left(E_{r}\right)$ and widths $(\Gamma)$ in $\mathrm{MeV}$ of low-lying resonances of ${ }^{11} \mathrm{~N}$.

\begin{tabular}{lcccccccc}
\hline \hline & \multicolumn{2}{c}{$N N+3 N 400$} & & \multicolumn{2}{c}{$\mathrm{N}^{2} \mathrm{LO}_{\text {sat }}$} & & \multicolumn{2}{c}{ Data evaluation [14] } \\
\cline { 2 - 3 }$J^{\pi}$ & $E_{r}$ & $\Gamma$ & & $E_{r}$ & $\Gamma$ & & $E_{r}$ & $\Gamma$ \\
\hline $1 / 2^{+}$ & 1.29 & 2.85 & & 1.33 & 1.45 & & $1.49(6)$ & $0.83(3)$ \\
$1 / 2^{-}$ & 1.91 & 0.54 & & 1.95 & 0.57 & & $2.22(3)$ & $0.6(1)$ \\
$5 / 2^{+}$ & 4.89 & 1.76 & & 3.81 & 0.53 & & $3.69(3)$ & $0.54(4)$ \\
$3 / 2^{-}$ & 4.62 & 0.47 & 4.60 & 0.70 & & $4.35(3)$ & $0.34(4)$ \\
$3 / 2^{+}$ & 5.88 & 4.09 & 4.39 & 2.55 & & $\ldots$ & $\ldots$ \\
$5 / 2^{-}$ & 5.85 & 0.66 & 4.77 & 0.41 & & \\
\hline \hline
\end{tabular}


though it provides the best fit of the present data angular distribution shape, is still missing a complete description of the nuclear force. This deficiency becomes apparent with this angular distribution data and is not possible to judge based on resonance energies alone.

We should, however, make it clear that the $\mathrm{N}^{2} \mathrm{LO}_{\text {sat }}$ interaction indeed captures some important missing physics compared to the other chiral interaction $(N N+3 N 400)$. It provides a more realistic description of the nuclear density and smaller gaps between major harmonic-oscillator shells. Overall, we observe that none of the available parametrizations of the chiral nuclear force are optimal in all aspects. There is significant progress in the development of high-quality chiral $N N$ potentials; the $\mathrm{N}^{4} \mathrm{LO}$ order has now been reached $[6,47]$. These potentials achieve an excellent description of the $N N$ system. However, despite this progress [48,49], a chiral $3 N$ force parametrization matching their quality is still missing.

In summary, with the measured angular distribution of low-energy elastic scattering off extremely exotic ${ }^{10} \mathrm{C}$ nuclei, we have demonstrated for the first time a strong sensitivity of this scattering to the nuclear force prescription. The low-level density and neutron-proton asymmetry in drip-line nuclei like ${ }^{10} \mathrm{C}$ bring in new and greater sensitivity to the nuclear force, allowing for discriminating between the different chiral interactions and finding further constraints for them. The measured ${ }^{10} \mathrm{C}(p, p){ }^{10} \mathrm{C}_{g s}$ differential cross section shows that only the $\mathrm{N}^{2} \mathrm{LO}_{\text {sat }}$ interaction provides an angular distribution shape consistent with the experiment but fails to reproduce its magnitude. This suggests that $\mathrm{N}^{2} \mathrm{LO}_{\text {sat }}$ is improved compared to the other forces but is still not an adequate description of the nuclear force. The new finding of this large sensitivity of the angular distribution will trigger more intensive efforts in ab initio calculations to single out which parameters and components of the chiral interactions are responsible for the successful description of the ${ }^{10} \mathrm{C}(p, p)$ data. Extreme systems, such as the ${ }^{11} N$ and ${ }^{10} \mathrm{C}(p, p)$ investigated here both experimentally and theoretically, thus provide one of the most stringent tests of the quality of the present and new generations of nuclear forces.

The authors express sincere thanks to the TRIUMF beam delivery team. The support from Canada Foundation for Innovation, NSERC, Nova Scotia Research and Innovation Trust, and the DFG through SFB 1245 is gratefully acknowledged. TRIUMF receives funding via a contribution through the National Research Council Canada. Computing support came from the LLNL institutional Computing Grand Challenge Program, from an INCITE Award on the Titan supercomputer of the Oak Ridge Leadership Computing Facility (OLCF) at ORNL, and from Calcul Quebec and Compute Canada. The work is prepared in part by LLNL under Contract No. DE-AC52-07NA27344. This material is based in part upon work supported by the U.S. Department of Energy, Office of Science, Office of Nuclear Physics, under Work Proposal No. SCW1158.

*ritu@triumf.ca

†navratil@triumf.ca

[1] S. Weinberg, Nucl. Phys. B363, 3 (1991).

[2] D. R. Entem and R. Machleidt, Phys. Rev. C 68, 041001 (2003).

[3] R. Machleidt and D. R. Entem, Phys. Rep. 503, 1 (2011).

[4] R. Roth, S. Binder, K. Vobig, A. Calci, J. Langhammer, and P. Navrátil, Phys. Rev. Lett. 109, 052501 (2012).

[5] A. Ekström, G. R. Jansen, K. A. Wendt, G. Hagen, T. Papenbrock, B. D. Carlsson, C. Forssén, M. Hjorth-Jensen, P. Navrátil, and W. Nazarewicz, Phys. Rev. C 91, 051301(R) (2015).

[6] E. Epelbaum, H. Krebs, and U.-G. Meißner, Phys. Rev. Lett. 115, 122301 (2015).

[7] S. C. Pieper and R. B. Wiringa, Annu. Rev. Nucl. Part. Sci. 51, 53 (2001).

[8] P. Navrátil, S. Quaglioni, I. Stetcu, and B. R. Barrett, J. Phys. G 36, 083101 (2009).

[9] B. R. Barrett, P. Navrátil, and J. P. Vary, Prog. Part. Nucl. Phys. 69, 131 (2013).

[10] J. Dilling and R. Krücken, Hyperfine Interact. 225, 111 (2014).

[11] G. C. Ball, G. Hackman, and R. Krücken, Phys. Scr. T 91, 093002 (2016).

[12] R. E. Laxdal et al., Nucl. Instrum. Methods Phys. Res., Sect. B 204, 400 (2003).

[13] R. Kanungo, Hyperfine Interact. 225, 235 (2014).

[14] J. H. Kelley, E. Kwan, J. E. Purcell, C. G. Sheu, and H. R. Weller, Nucl. Phys. A880, 88 (2012).

[15] V. Guimarães et al., Phys. Rev. C 67, 064601 (2003).

[16] See Supplemental Material at http://link.aps.org/ supplemental/10.1103/PhysRevLett.118.262502 for some details of the calculations and experiment, which includes Refs. [17-36].

[17] K. Wildermuth and Y.C. Tang, A Unified Theory of the Nucleus (Viewig+Teubner Verlag, Braunschweig, 1977).

[18] Y. C. Tang, M. LeMere, and D. R. Thompsom, Phys. Rep. 47, 167 (1978).

[19] T. Fliessbach and H. Walliser, Nucl. Phys. A377, 84 (1982).

[20] K. Langanke and H. Friedrich, in Advances in Nuclear Physics, edited by J. W. Negele and E. Vogt (Springer, New York, 1986), Chap. 17, pp. 223-363.

[21] P. Descouvemont and D. Baye, Rep. Prog. Phys. 73, 036301 (2010).

[22] M. Hesse, J.-M. Sparenberg, F. Van Raemdonck, and D. Baye, Nucl. Phys. A640, 37 (1998).

[23] M. Hesse, J. Roland, and D. Baye, Nucl. Phys. A709, 184 (2002).

[24] P. Navrátil, Few-Body Syst. 41, 117 (2007).

[25] F. Wegner, Ann. Phys. (Berlin) 506, 77 (1994).

[26] S. K. Bogner, R. J. Furnstahl, and R. J. Perry, Phys. Rev. C 75, 061001(R) (2007). 
[27] S. Szpigel and R. J. Perry, in Quantum Field Theory. A 20th Century Profile, edited by A. N. Mitra (Hindustan Publishing Co., New Delhi, 2000).

[28] G. Hupin, J. Langhammer, P. Navrátil, S. Quaglioni, A. Calci, and R. Roth, Phys. Rev. C 88, 054622 (2013).

[29] S. Binder, J. Langhammer, A. Calci, and R. Roth, Phys. Lett. B 736, 119 (2014).

[30] J. Langhammer, P. Navrátil, S. Quaglioni, G. Hupin, A. Calci, and R. Roth, Phys. Rev. C 91, 021301(R) (2015).

[31] E. Casarejos et al., Phys. Rev. C 73, 014319 (2006).

[32] K. Markenroth et al., Phys. Rev. C 62, 034308 (2000).

[33] A. Lépine-Szily et al., Phys. Rev. Lett. 80, 1601 (1998).

[34] J. M. Oliveira et al., Phys. Rev. Lett. 84, 4056 (2000).

[35] B. I. Schneider, Phys. Rev. A 24, 1 (1981).

[36] J. Dohet-Eraly, P. Navrátil, S. Quaglioni, W. Horiuchi, G. Hupin, and F. Raimondi, Phys. Lett. B 757, 430 (2016).

[37] S. Baroni, P. Navrátil, and S. Quaglioni, Phys. Rev. Lett. 110, 022505 (2013).

[38] S. Baroni, P. Navrátil, and S. Quaglioni, Phys. Rev. C 87, 034326 (2013).
[39] P. Navrátil, S. Quaglioni, G. Hupin, C. Romero-Redondo, and A. Calci, Phys. Scr. T 91, 053002 (2016).

[40] D. Gazit, S. Quaglioni, and P. Navrátil, Phys. Rev. Lett. 103, 102502 (2009).

[41] N. Kalantar-Nayestanaki, E. Epelbaum, J. G. Messchendorp, and A. Nogga, Rep. Prog. Phys. 75, 016301 (2012).

[42] H. Hergert, S. Binder, A. Calci, J. Langhammer, and R. Roth, Phys. Rev. Lett. 110, 242501 (2013).

[43] A. Cipollone, C. Barbieri, and P. Navrátil, Phys. Rev. Lett. 111, 062501 (2013).

[44] G. Hagen et al., Nat. Phys. 12, 186 (2016).

[45] R. Kanungo et al., Phys. Rev. Lett. 117, 102501 (2016).

[46] A. Calci, P. Navrátil, R. Roth, J. Dohet-Eraly, S. Quaglioni, and G. Hupin, Phys. Rev. Lett. 117, 242501 (2016).

[47] D. R. Entem, N. Kaiser, R. Machleidt, and Y. Nosyk, Phys. Rev. C 91, 014002 (2015).

[48] H. Krebs, A. Gasparyan, and E. Epelbaum, Phys. Rev. C 85, 054006 (2012)

[49] S. Binder et al., Phys. Rev. C 93, 044002 (2016). 